\title{
Microwave Synthesis of Zinc Oxide/Reduced Graphene Oxide Hybrid for Adsorption-Photocatalysis Application
}

\author{
Fatin Saiha Omar, ${ }^{1}$ Huang Nay Ming, ${ }^{1}$ Syed Muhamad Hafiz, ${ }^{1}$ and Lim Hong Ngee,3 \\ ${ }^{1}$ Department of Physics, Low Dimensional Materials Research Centre, Faculty of Science, University of Malaya, \\ 50603 Kuala Lumpur, Malaysia \\ ${ }^{2}$ Department of Chemistry, Faculty of Science, Universiti Putra Malaysia (UPM), 43400 Serdang, Selangor, Malaysia \\ ${ }^{3}$ Functional Device Laboratory, Institute of Advanced Technology, Universiti Putra Malaysia (UPM), 43400 Serdang, \\ Selangor, Malaysia
}

Correspondence should be addressed to Huang Nay Ming; huangnayming@gmail.com and Lim Hong Ngee; janet_limhn@yahoo.com

Received 4 November 2013; Accepted 9 December 2013; Published 2 January 2014

Academic Editor: Lizhi Zhang

Copyright (C) 2014 Fatin Saiha Omar et al. This is an open access article distributed under the Creative Commons Attribution License, which permits unrestricted use, distribution, and reproduction in any medium, provided the original work is properly cited.

\begin{abstract}
This work reports on synthesis of zinc oxide/reduced graphene oxide ( $\mathrm{ZnO} / \mathrm{rGO})$ nanocomposites in the presence of diethylenetriamine (DETA) via a facile microwave method. The X-ray diffraction (XRD) patterns of the nanocomposites correspond to the $\mathrm{ZnO}$ hexagonal phase wurtzite structure. The high-resolution transmission electron microscopy (HRTEM) images revealed that the $\mathrm{ZnO}$ nanorods, with an average length : diameter ratio of 10 , were successfully deposited on the rGO sheets. Under the irradiation of sunlight, the nanocomposites showed enhanced adsorption-photocatalysis by more than twofold and photocurrent response by sixfold compared to the $\mathrm{ZnO}$. The excellent photoactivity performance of the nanocomposites is contributed by smaller $\mathrm{ZnO}$ nanorod and the presence of $\mathrm{rGO}$ that acts as a photosensitizer by transferring electrons to the conduction band of $\mathrm{ZnO}$ within the nanocomposite during sunlight illumination.
\end{abstract}

\section{Introduction}

Direct discharge of pigments and dyes by textile industries into waters endangers the aquatic lives. The colours block the sunlight from passing through the water, causing disturbance to the natural growth cycles of the living organisms in the waters. The heavy metals and organic and inorganic complexes used in the making of pigments and dyes are highly toxic and will accumulate in the fat deposits of large fishes which will be consumed by organisms in the higher order on land. Conventional biological treatments are only effective to adsorb the dye, causing secondary pollution $[1,2]$.

Photocatalysis is a method used to eliminate organic compounds in wastewater by mineralizing them into the simplest compounds like water and carbon monoxide. Semiconductor photocatalysts have been studied extensively because of favorable combination of electronic structure, light absorption properties, and charge transport characteristics. $\mathrm{ZnO}$ has been known as a suitable alternative to $\mathrm{TiO}_{2}$ because of its strong oxidizing power, nontoxicity, and being relatively inexpensive. Its wide band gap $(3.37 \mathrm{eV})$ and higher electron mobility hamper its use as a photocatalyst [3-6].

In an effort to improve the photocatalytic efficiency of $\mathrm{ZnO}$, it has been doped, loaded, and combined with metals, nonmetals, and semiconductors [7-10]. Recently, researchers are astounded with graphene because of its unique electronic properties and large theoretical specific surface area. These properties make graphene a good candidate for combination with the $\mathrm{ZnO}$ because graphene's pristine mechanical performance stabilizes catalysis and offers a two-dimensional plane to deposit catalyst [11]. Transport through delocalized conjugated $\pi$ structures allows charge carriers in graphene to achieve high mobility and relatively slow charge recombination. Attempts to fabricate $\mathrm{ZnO} / \mathrm{rGO}$ composite have been reported to obtain superior properties [12-15]. In photocatalysis, $\mathrm{ZnO}$ can act as a photocatalyst while graphene 
acts as an electron-acceptor/transport material to facilitate the migration of photogenerated electrons and hinder the electron-hole recombination [16, 17]. Besides that, it has also been known to be an excellent adsorber for organic compounds [18].

In this work, a microwave approach was used to synthesize $\mathrm{ZnO} / \mathrm{rGO}$. Microwave radiation provides a fast and uniform heating rate that can cause rapid particles nucleation and growth which can reduce the reaction time, and therefore it can save a large amount of energy [19]. Diethylenetriamine (DETA) plays a role as a stabilizer by controlling the final morphology of the samples [20]. The defects in graphene oxide act as heterogeneous active sites for the nucleation and growth of $\mathrm{ZnO}$ particles [21]. The adsorption and photodegradation of methylene blue $(\mathrm{MB})$ and photocurrent of the $\mathrm{ZnO} / \mathrm{rGO}$ nanocomposites were investigated. Photocurrent is evidence to demonstrate the behavior of charge carriers within the samples under irradiation which can be correlated with the photocatalytic activity [22].

\section{Experimental}

2.1. Materials. Graphite flakes were purchased from Ashbury Inc. Zinc acetate dehydrate $\left(\mathrm{Zn}\left(\mathrm{CH}_{3} \mathrm{COO}\right)_{2} \cdot 2 \mathrm{H}_{2} \mathrm{O}\right)$ was purchased from Bendosen. Diethylenetriamine $\left(\mathrm{HN}\left(\mathrm{CH}_{2}\right.\right.$ $\left.\mathrm{CH}_{2} \mathrm{NH}_{2}\right)_{2}$ ) was purchased from Merck. Sodium hydroxide $(\mathrm{NaOH})$, potassium permanganate $\left(\mathrm{KMnO}_{4}\right)$, sulfuric acid $\left(\mathrm{H}_{2} \mathrm{SO}_{4}\right)$, and acid hydrochloric $(\mathrm{HCl})$ were purchased from R\&M Marketing. Hydrogen peroxide $\left(\mathrm{H}_{2} \mathrm{O}_{2}\right)$ and methylene blue were purchased from Systerm. Distilled water was used throughout the sample preparation.

\subsection{Methods}

2.2.1. Synthesis of $\mathrm{ZnO} / r G O$ Photocatalyst. Graphite powder was used as the starting reagent to synthesize GO using a simplified Hummer's method. $20 \mu \mathrm{L}$ of DETA was dropped into $4.6 \mathrm{mM}\left(\mathrm{Zn}\left(\mathrm{CH}_{3} \mathrm{COO}\right)_{2} \cdot 2 \mathrm{H}_{2} \mathrm{O}\right)$ solution and then was stirred for $30 \mathrm{~min}$. Certain amount of $14 \mathrm{mg} / \mathrm{mL}$ GO gel was sonicated in distilled water to produce uniform dispersion. Under stirring, the zinc solution was added dropwise into GO solution at a rate of a drop per $5 \mathrm{sec}$ followed by $0.09 \mathrm{M} \mathrm{NaOH}$ until reaching $\mathrm{pH}$ value of 12 and stirred for $13 \mathrm{~h}$. The mixture was then placed in a microwave and treated for $30 \mathrm{~min}$. After letting it cool at room temperature, the precipitates were washed three times with distilled water and finally dried in oven at $60^{\circ} \mathrm{C}$ for $24 \mathrm{~h}$. Three kinds of composite obtained with different GO loadings, $0.2,0.7$, and $1.5 \mathrm{wt} \%$ GO gel, were named as ZG3, ZG2, and ZG1, respectively. ZnO was synthesized using the similar procedure. For comparison purpose, composite without DETA was also prepared using the similar procedure and named as ZG.

2.3. Characterizations. The structure and morphology of the samples were characterized by X-ray diffractometry (XRD) Philips D5000 using CuK $\alpha$ radiation $(\lambda=1.4506 \AA$ ), field emission scanning electron microscope (FESEM) JSM$7600 \mathrm{~F}$, and high-resolution transmission electron microscope
(HRTEM) JEM-2100F. Ultraviolet-visible absorption spectra were recorded using UV-vis spectroscopy Thermo scientific Evolution 300. Raman spectra were recorded using a Renishaw inVia Raman microscope system.

2.4. Photocatalytic Experiments. The photocatalytic properties of the samples were evaluated by photodegradation of $\mathrm{MB}$ solution under natural sunlight. In every experiment, $5 \mathrm{mg}$ of photocatalyst was exposed to natural sunlight under magnetic stirring in $10 \mathrm{~mL}$ of $5 \mathrm{ppm} \mathrm{MB}$. Prior to irradiation, the suspensions were stirred in the dark overnight to achieve a saturated adsorption between the photocatalysts and $\mathrm{MB}$ molecules. The loaded samples were used repeatedly, and each cycle lasted for $2 \mathrm{~h}$. Before the next cycle, the remaining solution was replaced with fresh $\mathrm{MB}$ solution. Tow $\mathrm{mL}$ solution was withdrawn every $20 \mathrm{~min}$, and the concentration of MB solution was measured within $450 \mathrm{~nm}$ to $750 \mathrm{~nm}$ using a UV-Vis spectrometer. The change of relative absorbance was used to record the change of concentration of $\mathrm{MB}$ solution, that was $\mathrm{Ct} / \mathrm{Co}$ ( $\mathrm{Ct}$ referred to the concentration of $\mathrm{MB}$ solution at time $t$ and Co referred to the concentration of $\mathrm{MB}$ solution at initial time). All experiments were repeated six times.

2.5. Photocurrent Preparation. To investigate the transition of photogenerated electrons before and after the rGO loading, $\mathrm{ZnO}$ and $\mathrm{ZnO} / \mathrm{rGO}$ electrodes were prepared as follows: $0.5 \mathrm{mg}$ of the as-prepared photocatalyst was mixed in distilled water to produce slurry form, which was then smeared onto a $1 \mathrm{~cm} \times 1 \mathrm{~cm}$ indium-tin oxide (ITO) glass using doctor blade's method. The coated ITO glass was dried in the oven at $60^{\circ} \mathrm{C}$ for $24 \mathrm{~h}$. All investigated electrodes were of similar mass and exposed area.

The photocurrents were carried out using a VersaSTAT 3 potentiostat (Ametek Princeton Applied Research, Oak Ridge, TN). A solar light simulator Oriel Instrument was used to simulate sunlight under one sun AM 1.5 G with the measured light irradiance of $100 \mathrm{~mW} \mathrm{~cm} \mathrm{~cm}^{-2}$ provided by a $150 \mathrm{~W}$ xenon light source. A conventional three-electrode cell with working electrode (as-prepared photocatalyst), a platinum wire as counter electrode, an $\mathrm{Ag} / \mathrm{AgCl}$ (in $3 \mathrm{M} \mathrm{KCl}$ ) as reference electrode, and $1 \mathrm{M} \mathrm{KCl}$ as electrolyte was used. The photoresponse of the photocatalysts as light on and off were measured at $0.5 \mathrm{~V}$.

\section{Results and Discussion}

Figure 1 shows the XRD patterns of ZnO, ZG1, ZG2, ZG3, $\mathrm{rGO}$, and GO. All the diffraction peaks of $\mathrm{ZnO}$ are consistent with the hexagonal phase wurtzite structure (JCPDS number 80-0074). The peak (002) of GO at $11.1^{\circ}$ is ascribed to the introduction of oxygenated functional groups attached on both sides and edges of carbon sheets [23]. Meanwhile, the diffraction peaks of the nanocomposites are similar to those of $\mathrm{ZnO}$. The disappearance of the GO peak suggests the complete exfoliation of $\mathrm{GO}$ due to the insertion of $\mathrm{ZnO}$ particles [24]. No diffraction peak of rGO can be observed in the nanocomposites, which might be due to the low amount 


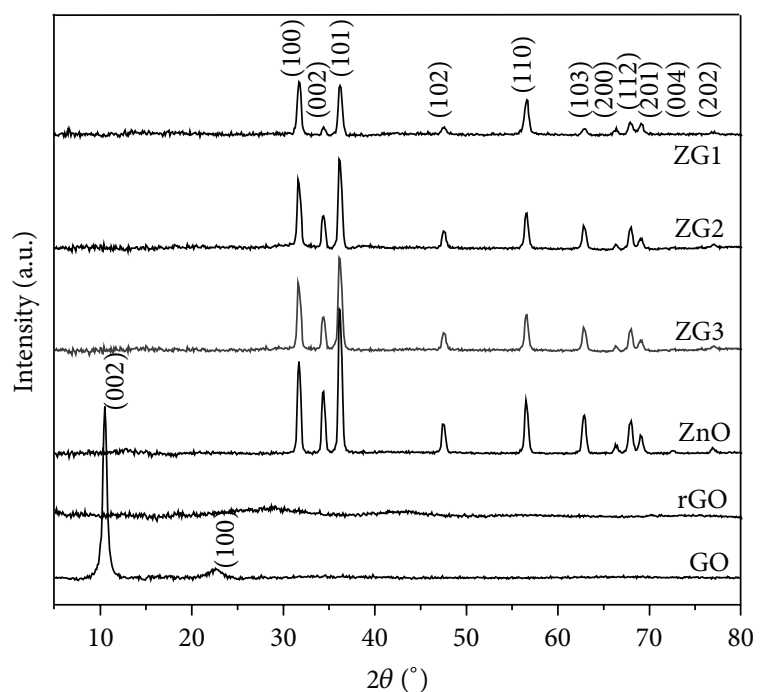

Figure 1: XRD patterns of GO, rGO, ZnO, ZG1, ZG2, and ZG3.

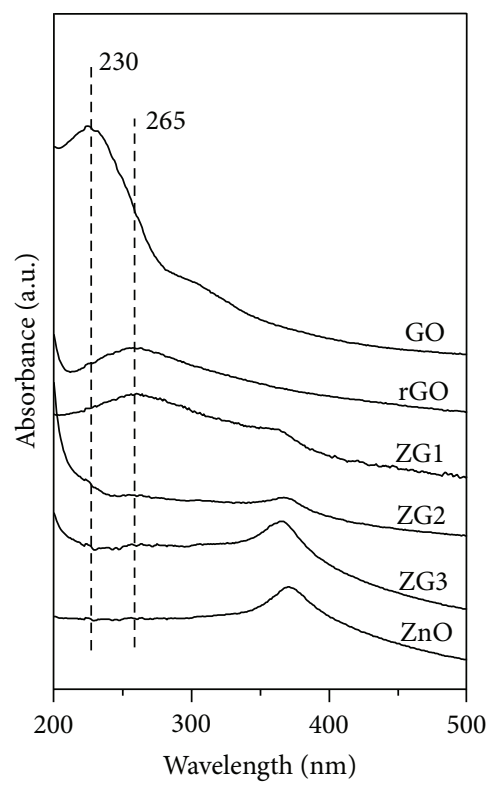

FIgURE 2: UV-Vis spectra of GO, rGO, ZnO, ZG1, ZG2, and ZG3.

and the extensive exfoliation of rGO. The reduction of GO is confirmed by the appearance of small bumps at $29^{\circ}$ and $44^{\circ}$, indicating the removal of a large number of oxygen-containing groups and the formation of much more disordered graphene sheets [25]. No other peak of impurity is detected.

Figure 2 shows the UV-Vis absorption spectra of $\mathrm{ZnO}$, ZG1, ZG2, ZG3, rGO, and GO. The spectra of GO exhibit a maximum absorption peak at $230 \mathrm{~nm}$, corresponding to the $\pi \rightarrow \pi$ transition of the aromatic $\mathrm{C}-\mathrm{C}$ bonds [26]. After the reduction of the GO, the absorption peak had red-shifted to $265 \mathrm{~nm}$, indicating restoration of $\pi$ conjugation within the rGO sheets [27]. The $\mathrm{ZnO}$ shows an absorption peak

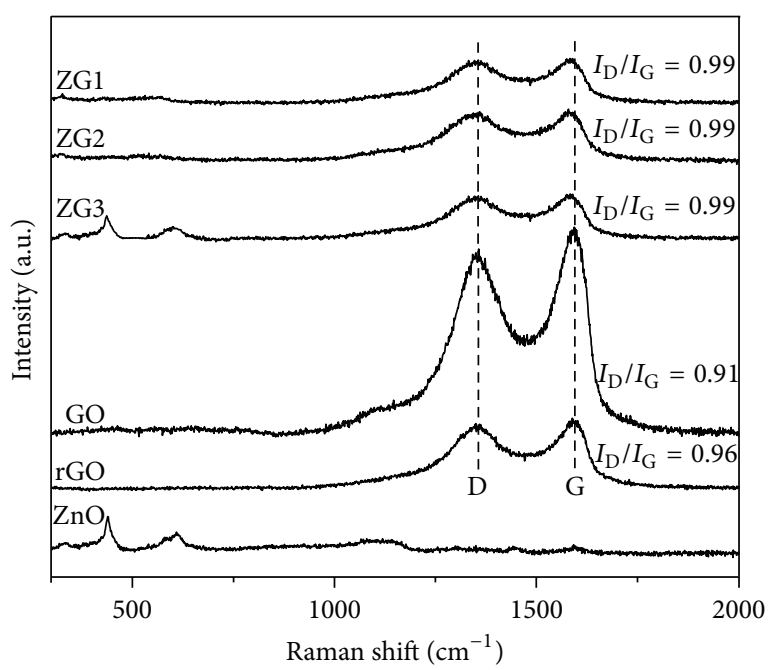

Figure 3: Raman spectra of GO, rGO, ZnO, ZG1, ZG2, and ZG3.

at $360 \mathrm{~nm}$ which can also be observed in the nanocomposites which suggests the presence of $\mathrm{ZnO}$ nanostructures [28]. Besides, the red shift of the $\mathrm{ZnO}$ absorption edge in the nanocomposites is ascribed to the chemical interaction between $\mathrm{ZnO}$ and rGO [29]. Comparing ZG1, ZG2, and ZG3, the relative intensity of the carbon peak $(\sim 265 \mathrm{~nm})$ increases with $\mathrm{rGO}$ loading in the nanocomposites.

The presence of both carbon and $\mathrm{ZnO}$ can be confirmed from the Raman spectra. In Figure 3, the spectrum for $\mathrm{ZnO}$ displays a peak at $330 \mathrm{~cm}^{-1}$ which is assigned to the secondorder Raman spectrum arising from zone-boundary phonons of hexagonal $\mathrm{ZnO}$. The intense peak at $440 \mathrm{~cm}^{-1}$ corresponds to E2 (HI) mode, which is the characteristic peak of the hexagonal wurtzite phase $\mathrm{ZnO}$. The peak at $582 \mathrm{~cm}^{-1}$ is assigned to $E 1$ (LO) mode, attributed to oxygen deficiency defects in $\mathrm{ZnO}$ [30]. The peak at $1130 \mathrm{~cm}^{-1}$ is due to the multiple-phonon scattering processes [31]. The intensity of these peaks is reduced in composites as compared to that in $\mathrm{ZnO}$ due to the interaction between $\mathrm{ZnO}$ and rGO.

The similar G and D band (where G peak refers to the presence of $\mathrm{sp}^{2}$ carbon-type structure and $\mathrm{D}$ peak refers to the presence of disorder in the graphene structure [32]) for rGO and nanocomposites suggests that the structure of rGO is maintained in the composites. The $\mathrm{D} / \mathrm{G}$ intensity value of the nanocomposites (0.99) increased compared with that of the GO (0.91) and rGO (0.96). The nanocomposite exhibits the highest $D / G$ value due to the increasing disorder of $\mathrm{sp}^{2}$ contributed by the presence of $\mathrm{ZnO}$ in the composites $[33,34]$. Compared with rGO, the D band was blue-shifted by $4 \mathrm{~cm}^{-1}$ while the $\mathrm{G}$ band was blue-shifted by $10 \mathrm{~cm}^{-1}$ in nanocomposites. These shifts are attributed to the chemical interaction between $\mathrm{ZnO}$ and $\mathrm{rGO}$ [35].

Figure 4(a) compares the Cls peak of GO and ZG1 nanocomposite. Both peaks can be deconvoluted into four Gaussian peaks, namely, $\mathrm{sp}^{2}$ carbon $(\mathrm{C}-\mathrm{C}, 284.5 \mathrm{eV}), \mathrm{C}-\mathrm{O}$ (286 eV), $\mathrm{C}=\mathrm{O}(287.4 \mathrm{eV})$, and $\mathrm{O}-\mathrm{C}=\mathrm{O}(288.8 \mathrm{eV})$. The peak intensity of the oxygen-bonded carbon reduced in the composite, indicating the reduction of GO after the microwave 


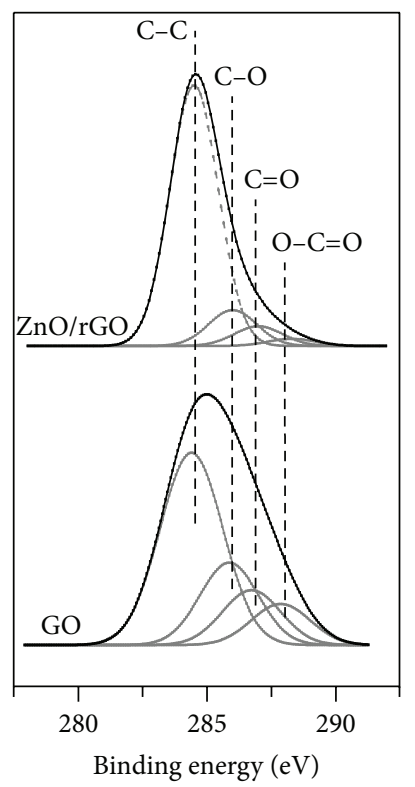

(a)

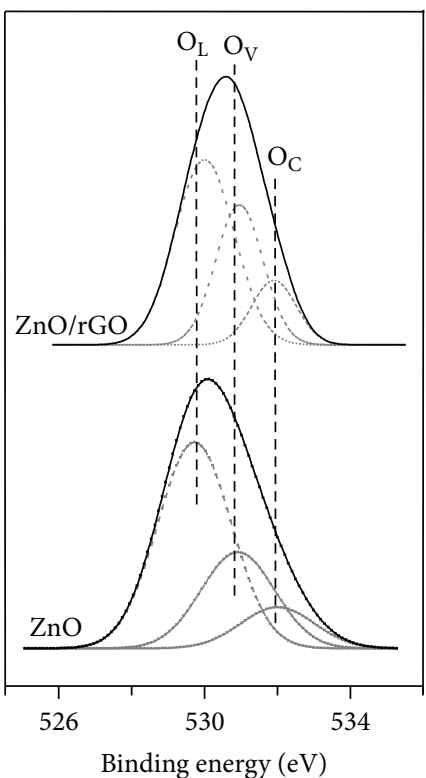

(b)

FIGURE 4: XPS spectra of (a) C1s of GO and ZG1 and (b) O1s of ZnO and ZG1.

treatment [36]. Figure 4(b) shows the O1s peak of $\mathrm{ZnO}$ and nanocomposite. The XPS pattern of $\mathrm{ZnO}$ can be deconvoluted into three Gaussian peaks, $\mathrm{O}_{\mathrm{L}}$ (oxygen lattice, $529.8 \mathrm{eV}$ ), $\mathrm{O}_{\mathrm{V}}$ (oxygen vacancy, $531 \mathrm{eV}$ ), and $\mathrm{O}_{\mathrm{C}}$ (chemisorbed oxygen species, $532.1 \mathrm{eV}$ ). The first two peaks are attributed to $\mathrm{O}^{2-}$ ion in the $\mathrm{Zn}-\mathrm{O}$ bonds and oxygen deficient defects, respectively. The last peak is assigned to the presence of loosely bound oxygen such as adsorbed $\mathrm{O}_{2}$ and $\mathrm{H}_{2} \mathrm{O}$ on the $\mathrm{ZnO}$ surface. The Ols peaks of the nanocomposite have slightly higher binding energies than $\mathrm{ZnO}$, with the deconvoluted peaks positioned at $530,531.2$, and $532.3 \mathrm{eV}$. Notice that the nanocomposite shows higher intensity on the second peak compared to $\mathrm{ZnO}$, indicating an increased density of oxygen vacancy on the $\mathrm{ZnO}$ surface. Oxygen vacancy could act as an electron trap that could hinder the electron-hole recombination and in turn could help in effective photocatalytic degradation [37].

As shown in Figure 5, individual $\mathrm{ZnO}$ and $\mathrm{ZnO}$ in the nanocomposites are rod-like with an average diameter and length of $100 \mathrm{~nm}$ and $1 \mu \mathrm{m}$, respectively. The nanocomposite in the absence of DETA (Figure 5(c)) shows that the size of the $\mathrm{ZnO}$ nanorod is bigger compared to the nanocomposite with DETA (Figure 5(b)). This is due to the interaction between $\mathrm{Zn}^{2+}$ with DETA that formed Zn-DETA complex. The ZnDETA complex hindered the fast reaction of $\mathrm{Zn}^{2+}$ with $\mathrm{OH}^{-}$, forming $\mathrm{ZnO}$ nanorods with smaller diameter [38].

Figure 6 revealed that, for $\mathrm{ZnO}$, the nanorods apparently originate from a centre, forming flower-like morphologies. Meanwhile, the $\mathrm{ZnO}$ rods are distributed on the surface of rGO for all the nanocomposites, confirming the interaction between $\mathrm{ZnO}$ and $\mathrm{rGO}$. The average diameter of the $\mathrm{ZnO}$ nanorods in the nanocomposite is slightly decreased with increasing rGO content due to the higher number of reaction sites provided by the GO that inhibited the agglomeration of $\mathrm{ZnO}$ particles [39]. The smaller size of the $\mathrm{ZnO}$ in the nanocomposites provides larger effective surface area in the enhancement of photocatalytic activity [40]. The crystal lattice fringes (Figure 6(e)) with a d-spacing of $0.26 \mathrm{~nm}$ can be assigned to the (002) plane of hexagonal $\mathrm{ZnO}$ [41].

\section{Photocatalysis}

Adsorption of the dye is an important parameter in determining the photodegradation rate. To distinguish the functions of the adsorption of $\mathrm{rGO}$ and photocatalysis of $\mathrm{ZnO}$, the suspension was stirred overnight in the dark to reach the saturated adsorption of $\mathrm{MB}$ before illumination.

Based on Figure 7(a), the $\mathrm{ZnO}$ shows 3\% of $\mathrm{MB}$ adsorption. When $\mathrm{rGO}$ is introduced to $\mathrm{ZnO}$, the adsorption rate increased to $45.7 \%, 64.9 \%$, and $82.3 \%$ for ZG3, ZG2, and ZG1, respectively. The enhancement of $\mathrm{MB}$ absorption increased with increasing proportion of rGO in the nanocomposites which is attributed to the $\pi-\pi$ stacking interactions between $\mathrm{MB}$ and rGO [42]. In Figure 7(b), the degradation rates for blank (without photocatalyst) ZG, ZnO, ZG3, ZG2, and ZG1 are $12 \%, 25 \%, 39.4 \%, 63.5 \%, 75.4 \%$, and $94.8 \%$, respectively. The observed enhanced photocatalytic activity by the nanocomposites is largely attributed to the smaller diameter of the $\mathrm{ZnO}$ nanorod providing larger effective surface area that improves the absorbance of $\mathrm{MB}$ and the presence of rGO that acts as a photosensitizer. rGO is photoexcited by sunlight and transfers electrons to the $\mathrm{CB}$ of $\mathrm{ZnO}$, raising the concentration of electrons leading to dye degradation [43].

The stability of photocatalyst during photocatalysis is a crucial factor for practical application. The reusability of ZG1 in photodegradation of $\mathrm{MB}$ is tested by repeating the photocatalytic experiment for six cycles after the completion of the photocatalytic experiment shown in Figure 7. In Figure $8(\mathrm{a}), \mathrm{MB}$ solution was almost completely adsorbed 


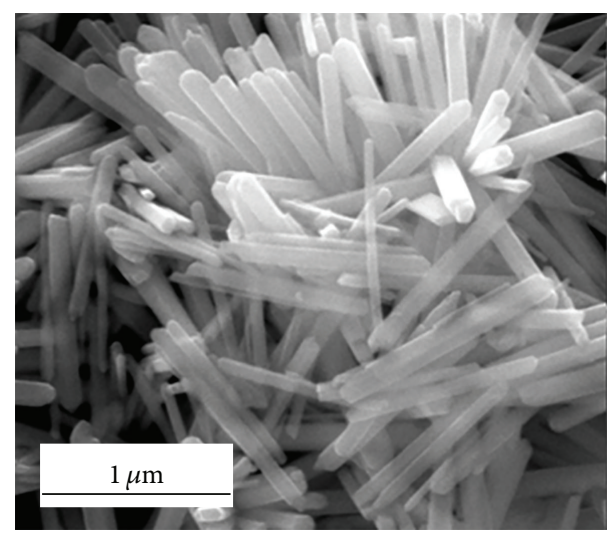

(a)

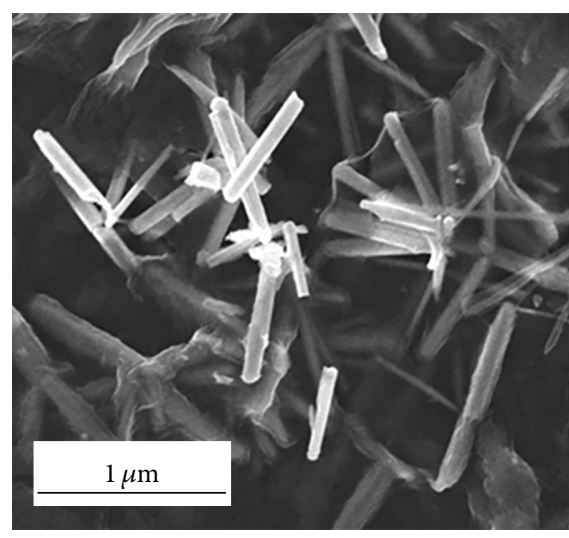

(b)

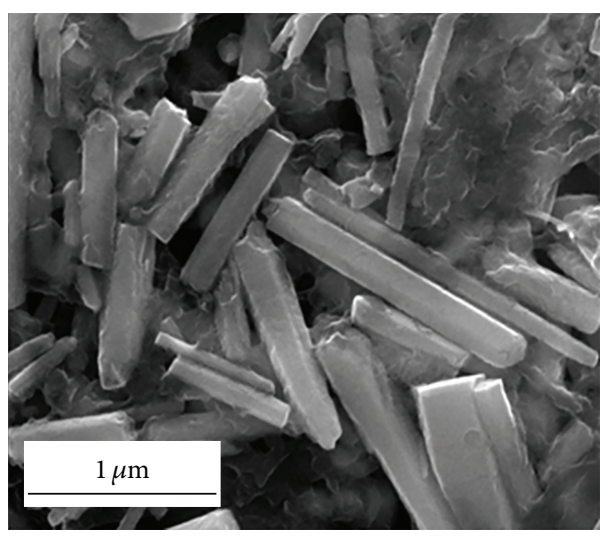

(c)

Figure 5: FESEM images of (a) ZnO, (b) ZG1, and (c) ZG.

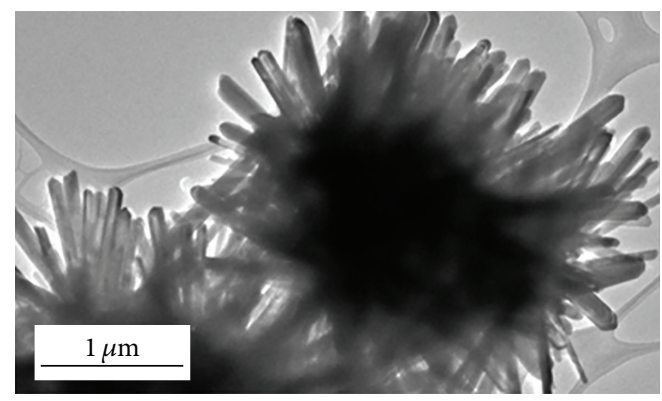

(a)

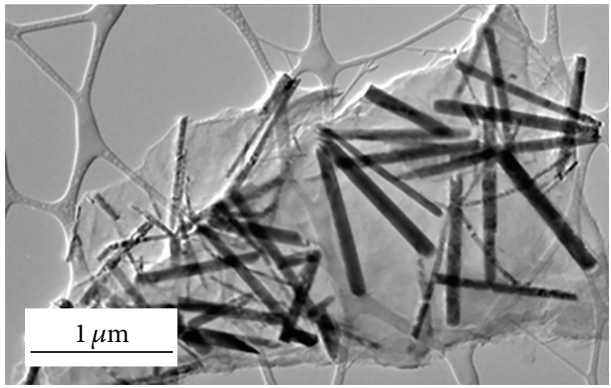

(c)

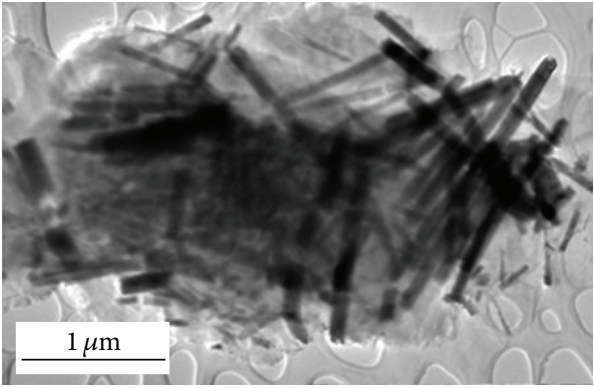

(b)

FIGURE 6: HRTEM images of (a) ZnO, (b) ZG3, (c) ZG2, (d) ZG1, and (e) ZG1 at a higher magnification. 


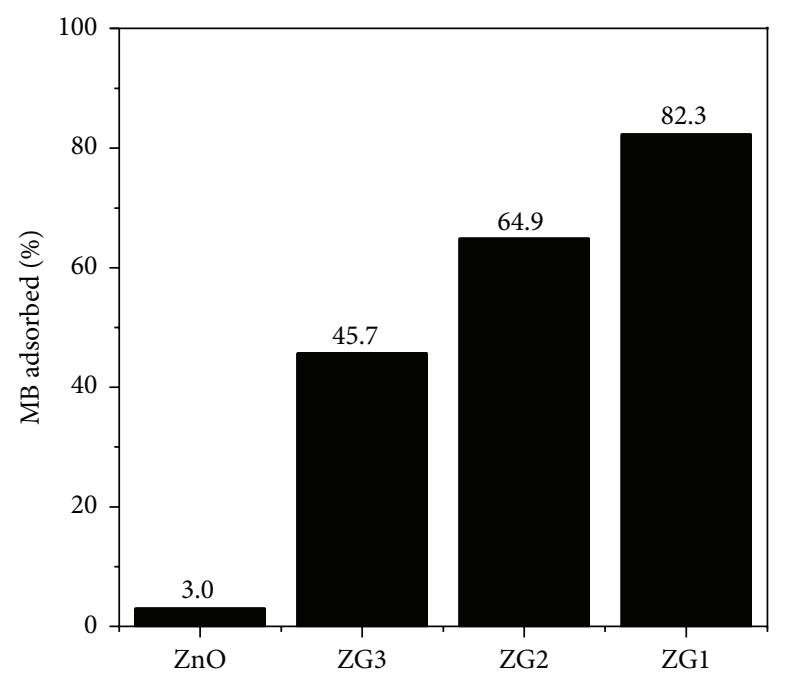

(a)

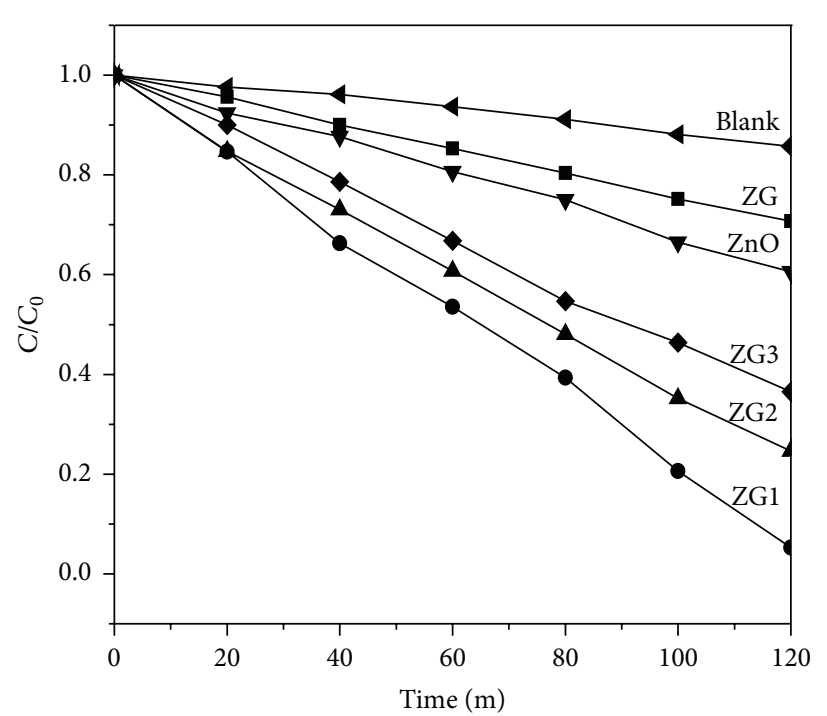

(b)

Figure 7: (a) Adsorption performance of ZnO, ZG1, ZG2, and ZG3 in the dark. (b) Photodegradation performance of blank (without photocatalyst) ZnO, ZG, ZG1, ZG2, and ZG3 under the sunlight.

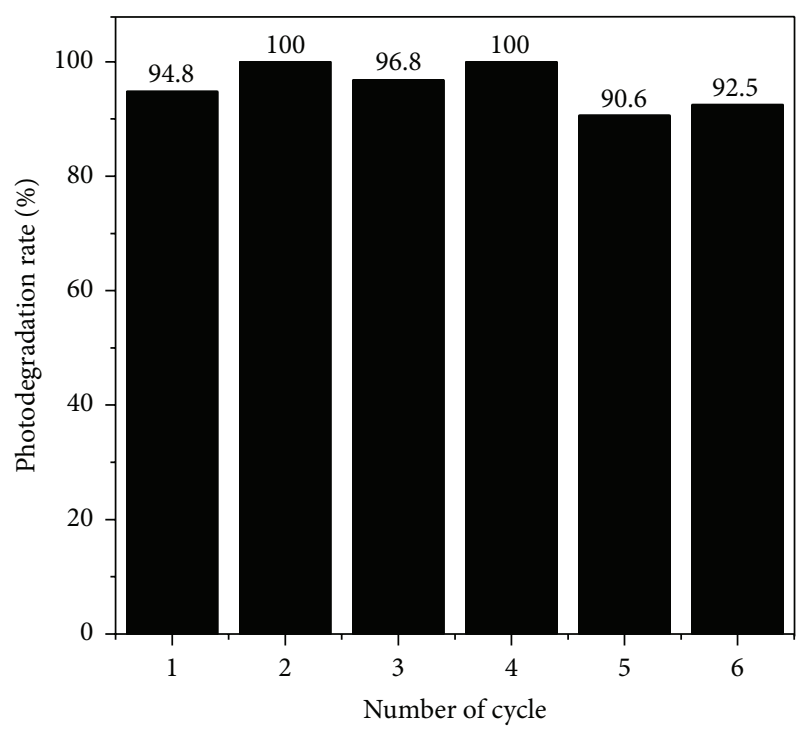

(a)

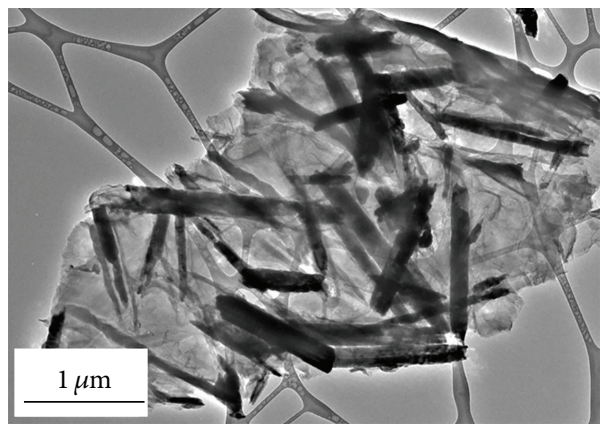

(b)

FIGURE 8: (a) Cycling runs in the photodegradation of MB by ZG1 under the sunlight. (b) HRTEM image of ZG1 for cycles.

and degraded by ZG1 for each cycle. ZG1 does not exhibit any changes in morphology even after the sixth cycle, revealing that it is photostable (Figure $8(\mathrm{~b})$ ).

4.1. Photocurrent. All the nanocomposites exhibit much higher photocurrent intensity than $\mathrm{ZnO}$, where the photocurrent of ZG1 is about eight times higher than that of $\mathrm{ZnO}$ (Figure 9). The photocurrents are $6.0 \mu \mathrm{A} \mathrm{cm}^{-2}, 7.5 \mu \mathrm{A} \mathrm{cm}^{-2}$, $11.0 \mu \mathrm{A} \mathrm{cm}^{-2}, 17.5 \mu \mathrm{A} \mathrm{cm}^{-2}$, and $24.7 \mu \mathrm{A} \mathrm{cm}^{-2}$ for $\mathrm{ZnO}, \mathrm{ZG}$, ZG3, ZG2, and ZG1, respectively. The generated photocurrent increased with the increase of the concentration of rGO, attributed to the extra electrons injection from the excited rGO within the nanocomposites under illumination [44].
These results are in agreement with the photocatalysis results (Figure 7(b)), where the rGO is photoexcited by sunlight, increasing the amount of electrons in the nanocomposite. This also shows that the nanocomposite could be applied as photoelectrochemical material for light-induced hydrogen evolution as photoinduced electrons and holes can participate in a redox reaction.

\section{Conclusions}

$\mathrm{ZnO} / \mathrm{rGO}$ nanocomposites were successfully synthesized via a facile microwave method in the presence of DETA. The stability of coordination structure between $\mathrm{Zn}^{2+}$ and DETA 


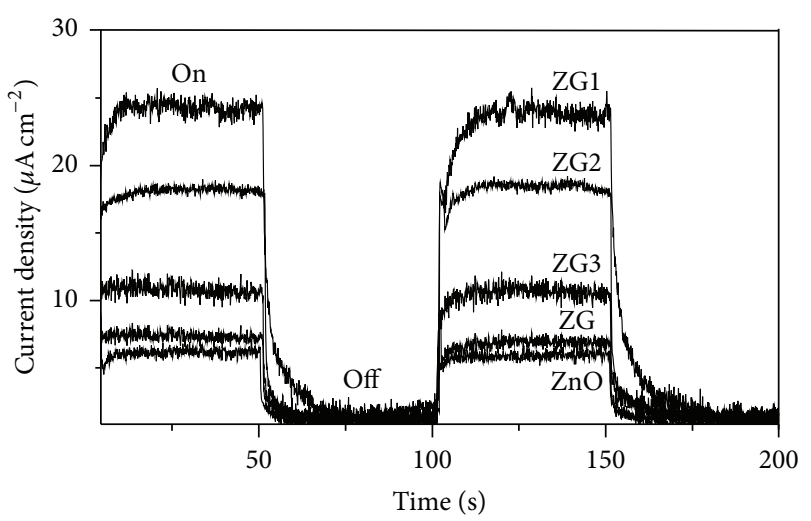

Figure 9: Photocurrent of ZnO, ZG, ZG1, ZG2, and ZG3 under solar light irradiation. $([\mathrm{KCl}]=1 \mathrm{M})$.

plays an important role for the final morphology of the $\mathrm{ZnO}$ and $\mathrm{ZnO} / \mathrm{rGO}$ nanocomposites. The adsorption and photocatalytic activities of the nanocomposites are dependent on the proportion of rGO loading in the samples. The nanocomposites outperformed $\mathrm{ZnO}$ due to synergistic effects of smaller $\mathrm{ZnO}$ nanorod and the presence of rGO that acts as a photosensitizer during sunlight illumination. It is also evident that the nanocomposite is stable as it displays consistent recycling for up to six runs. The nanocomposites show significantly higher photocurrent response than $\mathrm{ZnO}$, which is in agreement with the photocatalysis result, which manifest the possibility of using the nanocomposite for real application in wastewater treatment.

\section{Conflict of Interests}

The authors declare that there is no conflict of interests regarding the publication of this paper.

\section{Acknowledgments}

This work was supported by the High Impact Research Grant under the Ministry of Higher Education of Malaysia (UM.C/625/1/HIR/MOHE/05), L'Oréal Malaysia "For Women in Science Fellowships" 2011 (6375900-10501), and Postgraduate Research Fund (PG107-2012B).

\section{References}

[1] A. K. Asiagwu, "Sorption model for the removal of m-anisidine dye from aqueous solution using beaker's yeast (Saccharomuces cerevisiae)," International Journal of Research and Reviews in Applied Sciences, vol. 13, pp. 617-625, 2012.

[2] E. S. Beach, R. T. Malecky, R. R. Gil, C. P. Horwitz, and T. J. Collins, "Fe-TAML/hydrogen peroxide degradation of concentrated solutions of the commercial azo dye tartrazine," Catalysis Science and Technology, vol. 1, no. 3, pp. 437-443, 2011.

[3] A. K. Singh, S. S. Multani, and S. B. Patil, "ZnO nanorods and nanopolypods synthesized using microwave assisted wet chemical and thermal evaporation method," Indian Journal of Pure and Applied Physics, vol. 49, no. 4, pp. 270-276, 2011.
[4] M. Gusatti, J. D. A. do Rosário, C. E. M. de Campos et al., "Production and characterization of $\mathrm{ZnO}$ nanocrystals obtained by solochemical processing at different temperatures," Journal of Nanoscience and Nanotechnology, vol. 10, no. 7, pp. 4348-4351, 2010.

[5] S. Xu and Z. L. Wang, "One-dimensional ZnO nanostructures: solution growth and functional properties," Nano Research, vol. 4, no. 11, pp. 1013-1098, 2011.

[6] Y. Yang, L. Ren, C. Zhang, S. Huang, and T. Liu, "Facile fabrication of functionalized graphene sheets (FGS)/ZnO nanocomposites with photocatalytic property," ACS Applied Materials and Interfaces, vol. 3, no. 7, pp. 2779-2785, 2011.

[7] N. P. Mohabansi, V. B. Patil, and N. Yenkie, "A comparative study on photo degradation of methylene blue dye effluent by advanced oxidation process by using $\mathrm{TiO}_{2} / \mathrm{ZnO}$ photo catalyst," Rasayan Journal of Chemistry, vol. 4, no. 4, pp. 814-819, 2011.

[8] J. B. Zhong, J. Z. Li, X. Y. He et al., "Improved photocatalytic performance of Pd-doped ZnO," Current Applied Physics, vol. 12, no. 3, pp. 998-1001, 2012.

[9] M.-K. Lee and H.-F. Tu, "Au-ZnO and Pt-ZnO films prepared by electrodeposition as photocatalysts," Journal of the Electrochemical Society, vol. 155, no. 12, pp. D758-D762, 2008.

[10] J. V. Foreman, J. Li, H. Peng, S. Choi, H. O. Everitt, and J. Liu, "Time-resolved investigation of bright visible wavelength luminescence from sulfur-doped $\mathrm{ZnO}$ nanowires and micropowders," Nano Letters, vol. 6, no. 6, pp. 1126-1130, 2006.

[11] X. Zhou, T. Shi, and H. Zhou, "Hydrothermal preparation of $\mathrm{ZnO}$-reduced graphene oxide hybrid with high performance in photocatalytic degradation," Applied Surface Science, vol. 258, no. 17, pp. 6204-6211, 2012.

[12] A. R. Marlinda, N. M. Huang, M. R. Muhamad et al., "Highly efficient preparation of $\mathrm{ZnO}$ nanorods decorated reduced graphene oxide nanocomposites," Materials Letters, vol. 80, pp. 9-12, 2012.

[13] W. Zou, J. Zhu, Y. Sun, and X. Wang, "Depositing ZnO nanoparticles onto graphene in a polyol system," Materials Chemistry and Physics, vol. 125, no. 3, pp. 617-620, 2011.

[14] W.-T. Song, J. Xie, S.-Y. Liu et al., "Graphene decorated with $\mathrm{ZnO}$ nanocrystals with improved electrochemical properties prepared by a facile in situ hydrothermal route," International Journal of Electrochemical Science, vol. 7, no. 3, pp. 2164-2174, 2012.

[15] W. Zou, J. Zhu, and X. Wang, "Preparation and characterization of graphene oxide- $\mathrm{ZnO}$ nanocomposites," Materials Science Forum, vol. 688, pp. 228-232, 2011.

[16] T. Lv, L. Pan, X. Liu et al., "One-step synthesis of CdS-TiO chemically reduced graphene oxide composites via microwaveassisted reaction for visible-light photocatalytic degradation of methyl orange," Catalysis Science and Technology, vol. 2, no. 4, pp. 754-758, 2012.

[17] P. D. Tran, S. K. Batabyal, S. S. Pramana, J. Barber, L. H. Wong, and S. C. J. Loo, "A cuprous oxide-reduced graphene oxide $\left(\mathrm{Cu}_{2} \mathrm{O}-\mathrm{rGO}\right)$ composite photocatalyst for hydrogen generation: employing rGO as an electron acceptor to enhance the photocatalytic activity and stability of $\mathrm{Cu}_{2} \mathrm{O}$," Nanoscale, vol. 4, pp. 3875-3878, 2012.

[18] X. Dou, "Is graphene brand new in carbon-based semiconductor photocatalysts for organic pollutants degradation?" Journal of Thermodynamics and Catalysis, vol. 3, pp. 1-2, 2013.

[19] Y. Zheng, K. Lv, Z. Wang, K. Deng, and M. Li, "Microwaveassisted rapid synthesis of anatase $\mathrm{TiO}_{2}$ nanocrystals with 
exposed 001 facets," Journal of Molecular Catalysis A, vol. 356, pp. 137-143, 2012.

[20] W.-T. Yao, S.-H. Yu, S.-J. Liu, J.-P. Chen, X.-M. Liu, and F.-Q. $\mathrm{Li}$, "Architectural control syntheses of CdS and CdSe nanoflowers, branched nanowires, and nanotrees via a solvothermal approach in a mixed solution and their photocatalytic property," Journal of Physical Chemistry B, vol. 110, no. 24, pp. 11704-11710, 2006.

[21] X. Xu, Y. Zhou, T. Yuan, and Y. Li, "Methanol electrocatalytic oxidation on Pt nanoparticles on nitrogen doped graphene prepared by the hydrothermal reaction of graphene oxide with urea," Electrochimica Acta, vol. 112, pp. 587-595, 2013.

[22] Y. Liu, C. Xie, J. Li, T. Zou, and D. Zeng, "New insights into the relationship between photocatalytic activity and photocurrent of $\mathrm{TiO}_{2} / \mathrm{WO}_{3}$ nanocomposite," Applied Catalysis A, vol. 433434, pp. 81-87, 2012.

[23] Y. Li, W. Gao, L. Ci, C. Wang, and P. M. Ajayan, "Catalytic performance of Pt nanoparticles on reduced graphene oxide for methanol electro-oxidation," Carbon, vol. 48, no. 4, pp. 11241130, 2010.

[24] S. V. Kumar, N. M. Huang, N. Yusoff, and H. N. Lim, "High performance magnetically separable graphene/zinc oxide nanocomposite," Materials Letters, vol. 93, pp. 411-414, 2013.

[25] G. Singh, A. Choudhary, D. Haranath et al., "ZnO decorated luminescent graphene as a potential gas sensor at room temperature," Carbon, vol. 50, no. 2, pp. 385-394, 2012.

[26] Y. K. Kim and D. H. Min, "Simultaneous reduction and functionalization of graphene oxide by polyallylamine for nanocomposite formation," Carbon Letters, vol. 13, pp. 29-33, 2012.

[27] J. Yang and S. Gunasekaran, "Electrochemically reduced graphene oxide sheets as high performance supercapacitors," Carbon, vol. 51, pp. 36-44, 2013.

[28] Y. Yang and T. Liu, "Fabrication and characterization of graphene oxide/zinc oxide nanorods hybrid," Applied Surface Science, vol. 257, no. 21, pp. 8950-8954, 2011.

[29] X. Liu, L. Pan, Q. Zhao et al., "UV-assisted photocatalytic synthesis of $\mathrm{ZnO}$-reduced graphene oxide composites with enhanced photocatalytic activity in reduction of $\mathrm{Cr}(\mathrm{VI})$," Chemical Engineering Journal, vol. 183, pp. 238-243, 2012.

[30] J. Y. Li and H. Li, "Physical and electrical performance of vapor-solid grown $\mathrm{ZnO}$ straight nanowires," Nanoscale Research Letters, vol. 4, no. 2, pp. 165-168, 2009.

[31] X.-Y. Ye, Y.-M. Zhou, Y.-Q. Sun, J. Chen, and Z.-Q. Wang, "Preparation and characterization of $\mathrm{Ag} / \mathrm{ZnO}$ composites via a simple hydrothermal route," Journal of Nanoparticle Research, vol. 11, no. 5, pp. 1159-1166, 2009.

[32] A. C. Ferrari, "Raman spectroscopy of graphene and graphite: disorder, electron-phonon coupling, doping and nonadiabatic effects," Solid State Communications, vol. 143, no. 1-2, pp. 47-57, 2007.

[33] S. Stankovich, D. A. Dikin, R. D. Piner et al., "Synthesis of graphene-based nanosheets via chemical reduction of exfoliated graphite oxide," Carbon, vol. 45, no. 7, pp. 1558-1565, 2007.

[34] J. Yang, X. Zhao, X. Shan et al., "Blue-shift of UV emission in $\mathrm{ZnO/graphene} \mathrm{composites,"} \mathrm{Journal} \mathrm{of} \mathrm{Alloys} \mathrm{and} \mathrm{Compounds,}$ vol. 556, pp. 1-5, 2013.

[35] T. Xu, L. Zhang, H. Cheng, and Y. Zhu, "Significantly enhanced photocatalytic performance of $\mathrm{ZnO}$ via graphene hybridization and the mechanism study," Applied Catalysis B, vol. 101, no. 3-4, pp. 382-387, 2011.
[36] H. Kim, J. T. Baek, and H. H. Park, "A study of the electrical properties of graphene-incorporated direct-patternable $\mathrm{ZnO}$ thin films," Thin Solid Films, vol. 529, pp. 234-2237, 2013.

[37] A. Prakash, S. K. Misra, and D. Bahadur, "The role of reduced graphene oxide capping on defect induced ferromagnetism of ZnO nanorods," Nanotechnology, vol. 24, Article ID 095705, 2013.

[38] J. Wang, B. Li, J. Chen et al., "Diethylenetriamine-assisted synthesis of CdS nanorods under reflux condition and their photocatalytic performance," Journal of Alloys and Compounds, vol. 535, pp. 15-20, 2012.

[39] H. N. Lim, R. Nurzulaikha, I. Harrison et al., "Preparation and characterization of tin oxide, $\mathrm{SnO}_{2}$ nanoparticles decorated graphene," Ceramics International, vol. 38, no. 5, pp. 4209-4216, 2012.

[40] N. Xu, Z. Shi, Y. Fan, J. Dong, J. Shi, and M. Z.-C. Hu, "Effects of particle size of $\mathrm{TiO}_{2}$ on photocatalytic degradation of methylene blue in aqueous suspensions," Industrial and Engineering Chemistry Research, vol. 38, no. 2, pp. 373-379, 1999.

[41] F. Ye, Y. Peng, C. Guang-Yi, B. Deng, and X. An-Wu, "Facile solution synthesis and characterization of $\mathrm{ZnO}$ mesocrystals and ultralong nanowires from layered basic zinc salt precursor," Journal of Physical Chemistry C, vol. 113, no. 24, pp. 10407-10415, 2009.

[42] R. Kou, Y. Shao, D. Wang et al., "Enhanced activity and stability of Pt catalysts on functionalized graphene sheets for electrocatalytic oxygen reduction," Electrochemistry Communications, vol. 11, no. 5, pp. 954-957, 2009.

[43] A. A. Más and D. Wei, "Photoelectrochemical properties of graphene and its derivatives," Nanomaterials, vol. 3, pp. 325356, 2013.

[44] J. Durantini, P. P. Boix, M. Gervaldo et al., "Photocurrent enhancement in dye-sensitized photovoltaic devices with titania-graphene composite electrodes," Journal of Electroanalytical Chemistry, vol. 683, pp. 43-46, 2012. 

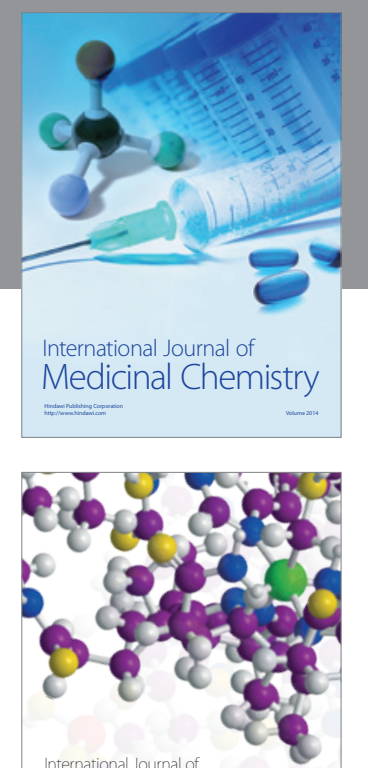

\section{Carbohydrate} Chemistry

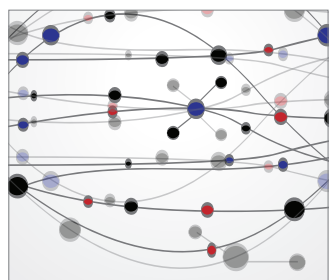

The Scientific World Journal
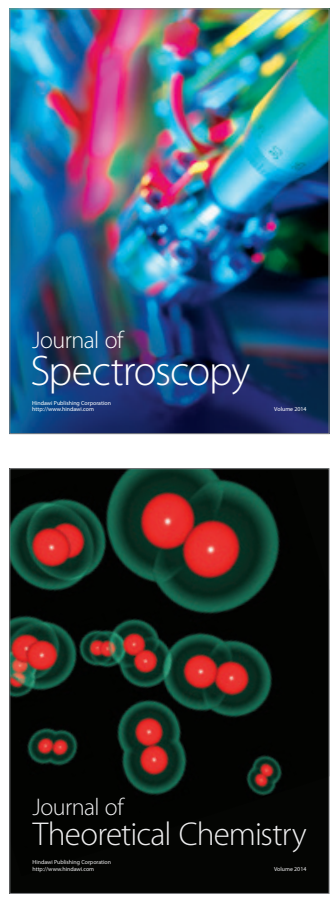
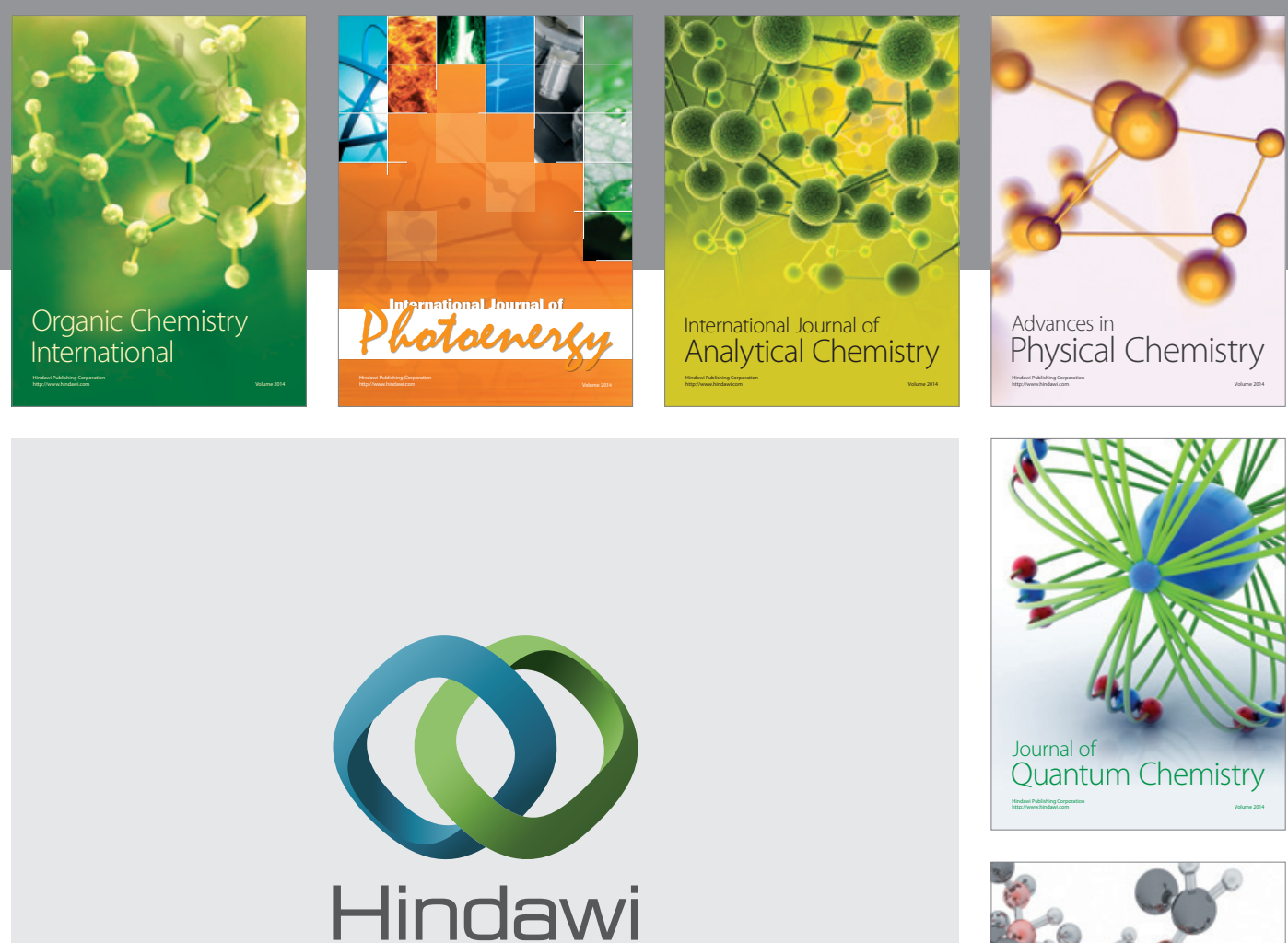

Submit your manuscripts at

http://www.hindawi.com

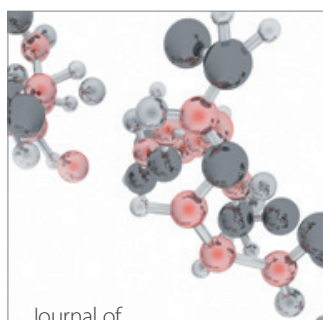

Analytical Methods

in Chemistry

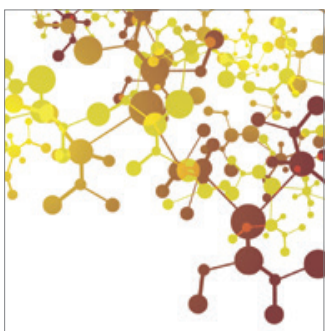

Journal of

Applied Chemistry

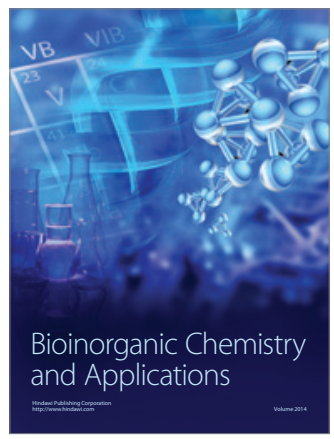

Inorganic Chemistry
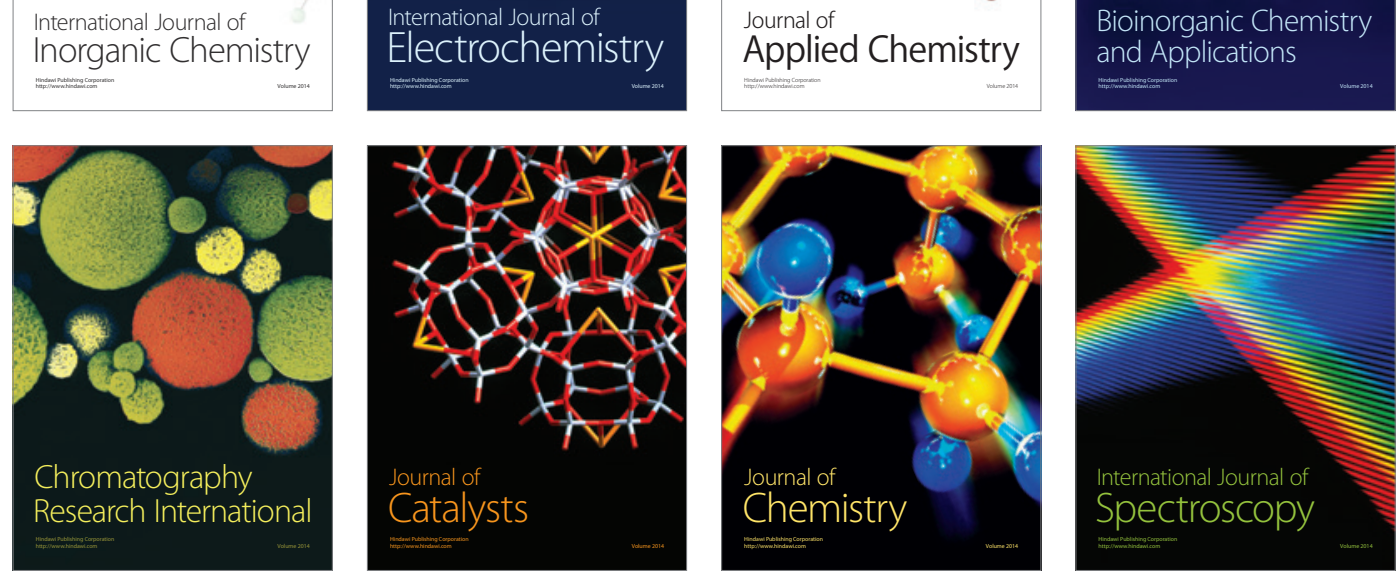